\title{
Micro-\& Nano-characterizations of Nanocrystalline CdS Thin Films from a Continuous Impinging Flow Microreactor
}

\author{
P. H. Mugdur*, A.A. Morrone**, S-.Y. Han****, Y.-J. Chang*, S. O. Ryu****, T-J. \\ Lee***, and C.-H. Chang* \\ *Department of Chemical Engineering, Oregon State University, Corvallis OR 97331 \\ **Seagate Technology, Minneapolis MN 55435-5489 \\ *** School of Chemical Engineering and Technology, Yeungnam University, Kyongsan, \\ 712-749 Korea
}

Chemical Bath Deposition (CBD) has been primarily used in the process of fabricating $\mathrm{Cu}$ (In, $\mathrm{Ga}$ ) $\mathrm{Se}_{2}$ and $\mathrm{CdTe}$ based thin film photovoltaics. Normally carried out as a batch process, the most significant feature of $\mathrm{CBD}$ is its ability to deposit thin films at low temperature. However, a major drawback of CBD is the formation of particles which generate waste and create defects in devices. Moreover, it is necessary to better understand the role of particle formation and deposition in CBD thin film growth process. We have developed a continuous impinging flow microreactor for CBD. This novel reactor provides the capability to control the particle size and size distribution before impinging on the substrate and thus helps us in differentiating the molecule-by-molecule growth from the cluster-by-cluster growth. It can also be used to deposit compound semiconductor thin films for electronic devices with improved film quality and minimum waste production. Our continuous impinging flow microreactor setup makes use of a micromixer for efficient mixing of the reactant streams.

CdS semiconductor thin films were successfully deposited on oxidized silicon substrates using this novel microreactor setup. In this study, comparisons of nanostructured thin films deposited by a batch reactor and a continuous impinging flow microreactor were performed by various characterization techniques. The surface morphology of the deposited films, carried out by AFM, SEM and Dektak surface profiler, clearly indicated an improved film quality from the microreactor. As seen in Fig. 1a, the microreactor produced a dense and uniform nanocrystalline CdS film. On the other hand, the batch reactor produced groups of non-continuous $\mathrm{CdS}$ nanocrytals on the substrate with the same amount of time (Fig. 1b). The CBD solutions were characterized by collecting samples at certain time using TEM copper grids covered with thin lacey carbon film. No nanoparticles could be observed from the impinging flow solution. In contrast, the batch solution produced agglomerates of nanoparticles over $0.1 \mathrm{um}$ in size with individual particles up to $10 \mathrm{~nm}$ in diameter as shown in Fig. 2.

This work is supported by the National Science Foundation's Process \& Reaction Engineering program under a CAREER grant \# CTS-0348723. The authors like to acknowledge Dr. Phil Watson for his assistance in AFM measurements. 

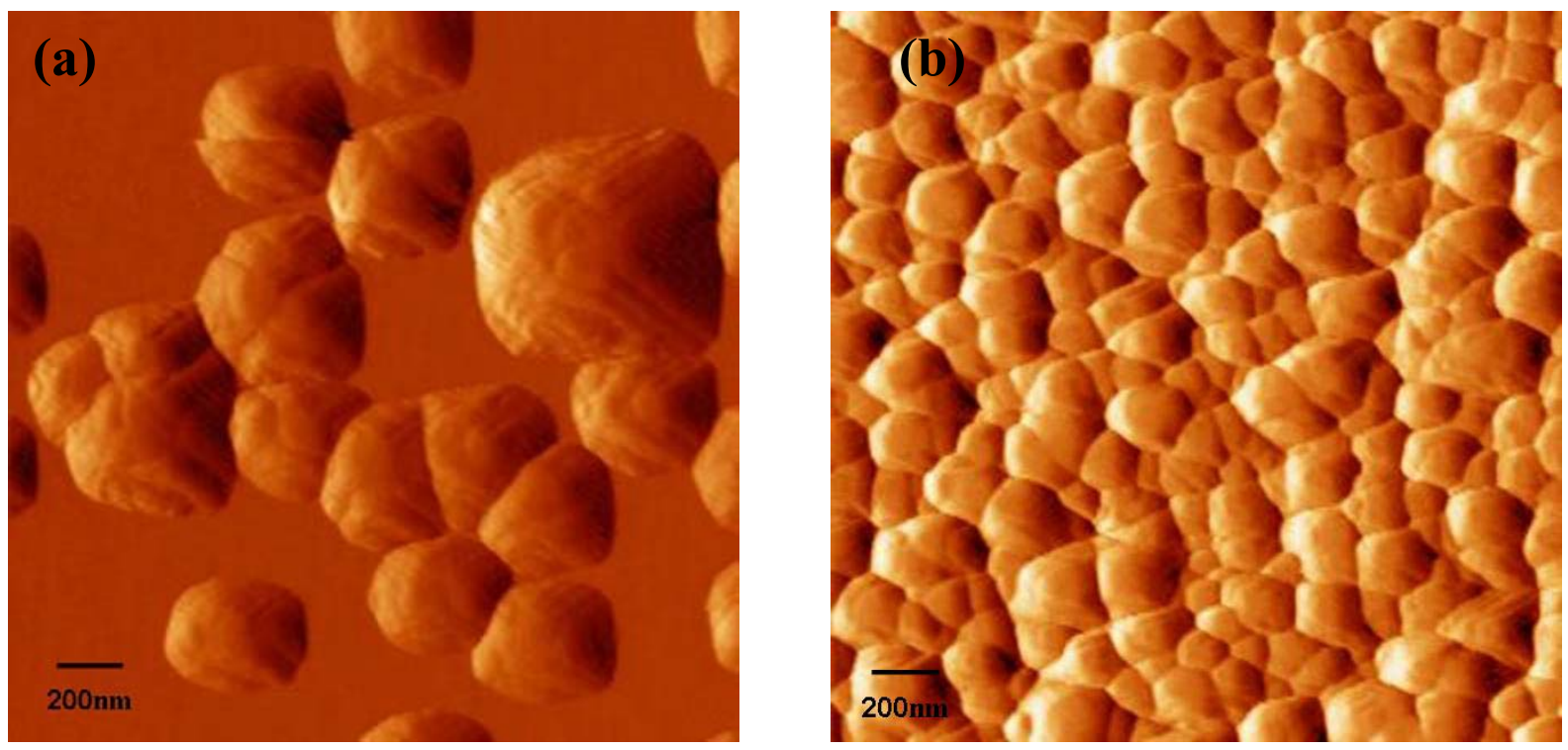

Fig.1. Comparison of AFM images of CdS films deposited by (a) batch and (b) continuous flow reactor.

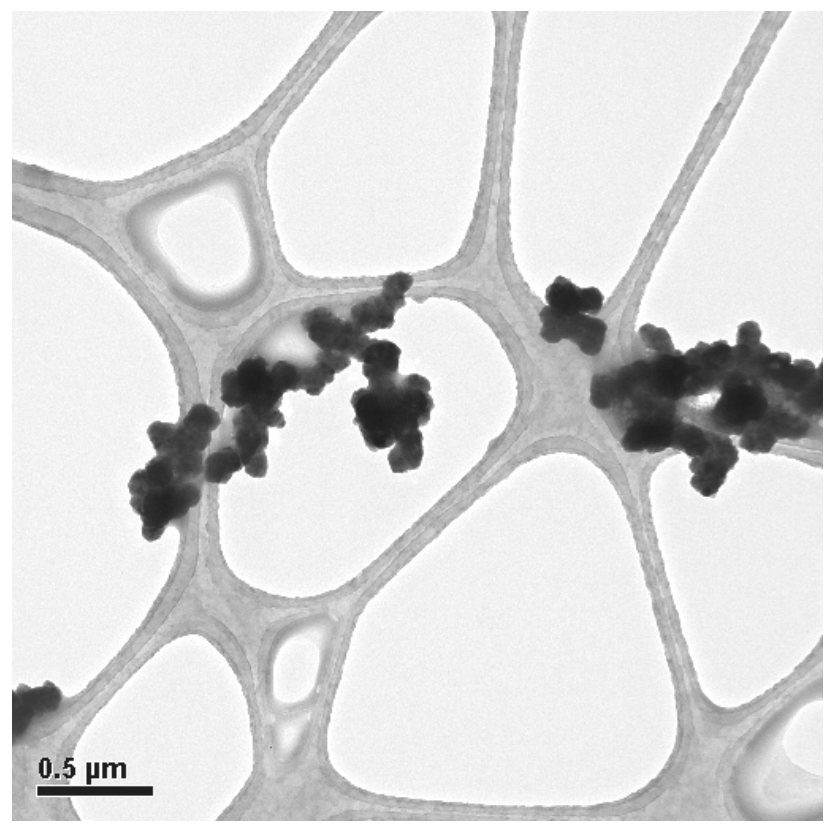

Fig.2. TEM image of CdS from batch process

(with reaction time $\mathrm{t}=3.12 \mathrm{~min}$ ) showing agglomerates like bunch of grapes. 